\title{
Characterizations and Criteria of Rupture of the Fibrous Composite Structures
}

\author{
Salah Mohammed Alsubari \\ Faculty of Education and Languages \\ Department of mathematics, Amran University \\ Republic of Yemen
}

\begin{abstract}
In this paper, we propose to study the simple case of the laminated composite plates, the criteria of rupture are applied to the various layers taken individually. Indeed, the resistance of a laminated structure is primarily based on the individual resistance of each layer.

When the requests increase, we observe successive ruptures of the layers and this of the first rupture of the first layer until the rupture of the last layer which coincides with the final rupture of the structure. We noted that the constraints are calculated by the Sinus model of the plates, developed by the finite element method. The results which we obtained by our calculations with the results of reference do not show significant differences which have the advantage of the facility of handling compared to the numerical method of the finite elements.
\end{abstract}

\section{Keywords}

Laminated composite plates, rupture of laminated structures, criteria of rupture

\section{INTRODUCTION}

The characterization of the resistance of the composites, and in particular laminated plates, requires determination of the constraints responsible for their damages within the framework of the criteria of ruptures based on the assumption of the homogeneity of the medium, at least finside the layers of the laminate.

Indeed, criteria of Irupture are the support of calculations of the structures. They can be applied formally to the various states of definitions of the limiting resistance of the studied composite structures.

In the case of the laminated composite plates, the criteria of rupture are applied to the various layers taken individually.

Indeed, the resistance of a laminated structure is primarily based on the individual resistance of each layer.

When the requests increase, one observes successive ruptures of the layers and this of the first rupture of the first layer until the rupture of the last layer which coincides with the final rupture of the structure.

It is obvious that with each rupture of layer the behavior mechanics global of the laminate are modified and its rigidity decreases. It is significant to note that the beginning of the damage of each layer depends its thickness and on the orientation of fibres in this layer compared to the load applied.

As an example, in the case of a laminate $(0, \pm 45, \pm 90)$ made up of layers of the same thickness and subjected to a traction in direction 0 , the first cracking appear in layers \pm 90 then in layers \pm 45 .
Lastly, the third phase is marked by the appearance of cracks in layers 0 . These cracks generate a delamination of the layers followed by the rupture of the layers to \pm 90 , then layers with \pm 45 , and finally the rupture of fibres in the layers with 0 , leading to the final rupture of the laminate.

However, the techniques of micromechanical analysis which allow the follow-up of the mechanisms of the rupture of the laminated structures are very complex and it is difficult to incorporate them in the tools of the design and analysis of the composite structures $[20,21,23]$.On the other hand, the analysis of the mechanical resistance is done by using the global aspect of the rupture. Generally, the criteria of rupture are based on the theory of Von Mises .

These criteria are established in the case of an individual layer and can being classified following the criteria of the maximum constraint, the maximum deformation or following the energy, interactive criteria.

\section{CRITERIA OF RUPTURE}

The tools of the modelling of the behaviour mechanics and calculations of the structures make it possible to determine the constraints in the layers of the laminated structures, as well as the constraints of interactions of the layers, which can be considered in the composite structural design.

However, the analysis of the concentration of the constraints and particularly the analysis of delamination are more complexes in the composite structures compared to isotropic materials. Indeed, the constraints of interfaces are strongly nonlinear compared to the thicknesses and the orientation of fibres. The formulations which result some are not easily usable, as parameters of optimization.

In the literature we meets several criteria of rupture to determine the state of limiting load [1], [6, 10,12, 15]. IThe first work goes back to 1864 with Tresca and its criterion of the maximum shear stress. IThe isotropic law of flow was formulated by Saint - Coming and Levy (1871), but it was necessary to wait the Fifties to see of it a use in the problems of the composite structures.

One of the first criteria of rupture applied to composite materials was introduced by Hill [2] [8,22], who proposes a generalization of the criterion of Von Mises by balancing each term by a coefficient to introduce the anisotropy of the equivalent medium. It considers a particular anisotropy which preserves three symmetry planes in the state of work hardening of material. The interactions of these three plans are the principal axes of anisotropy, which are not inevitably the principal directions of the constraints.

The criterion is formulated by saying that the absolute limit of the constraints corresponding to the rupture of material occurs by the following expression: 
$F\left(\sigma_{11}-\sigma_{22}\right)^{2}+G\left(\sigma_{22}-\sigma_{33}\right)^{2}$

$+H\left(\sigma_{33}-\sigma_{11}\right)^{2}$

$+2 L \sigma_{12}^{2}+2 M \sigma_{23}^{2}+2 N \sigma_{13}^{2}=1$

The coefficients scalar F, G, H, L, M and N are connected to the limiting values of the constraints according to the three directions and can be given using three experiments of traction and three experiments of simple shearing.

The improvement of the criterion of Hill was carried out by Hoffman [ 3] [7, 16 , 18, 19] which considered the linear terms of the normal constraints to take into account the dissymmetrical behaviors of materials

This makes it possible to introduce resistances of compression and traction by supposing a symmetrical behavior shearing. That is well checked in experiments. Thus the criterion is expressed in the form of a quadratic relation which is written:

$$
\begin{aligned}
& C_{1}\left(\sigma_{11}-\sigma_{22}\right)^{2}+C_{2}\left(\sigma_{22}-\sigma_{33}\right)^{2} \\
& +C_{3}\left(\sigma_{33}-\sigma_{11}\right)^{2} \\
& +C_{4} \sigma_{12}^{2}+C_{5} \sigma_{23}^{2}+C_{6} \sigma_{13}^{2} \\
& +C_{7} \sigma_{11}^{2}+C_{8} \sigma_{22}^{2}+C_{9} \sigma_{33}^{2}=1
\end{aligned}
$$

The coefficients $C_{i}$ are in experiments given, according to the ultimate stresses of traction, compression and shearing. While being based on the criterion of Hoffman, Tsai and $\mathrm{Wu}$ [ $4,5,617,21]$ proposed to introduce tensors of resistance of second and fourth order, in order to improve the correlation between the theoretical and experimental results.

The general formulation of the criterion of rupture is written in a polynomial form:

$$
F_{i} \sigma_{i}+F_{i j} \sigma_{i} \sigma_{j}+F_{i j k} \sigma_{i} \sigma_{j} \sigma_{k}=1
$$

$i, j, k=1,2,3 \ldots \ldots, 6$

The assumptions of orthotropic of composite materials with,one-way fibres, involve a reduction of Inumbers coefficients of the tensor of resistance $\left(F_{i}, F_{i j}, F_{i j k}\right)$ from 27 to 12 coefficients, while writing:

$$
F_{i j}=F_{j i} F_{i j k}=F_{i j k}=F_{j k i}=F_{k j i}
$$

we ww we are noted that in practice the simplified form of this criterion which is retained is:

$$
F_{i} \sigma_{i}+F_{i j} \sigma_{i} \sigma_{j}=1
$$

The principal coefficients $\left(F_{i}, F_{i i}\right)$ can be determined by compression and tensile tests according to various directions. The coefficients of $F_{i j}$ interactions can be determined by bi axial tests, provided that the proportion of the constraints is well controlled.

\section{CRITERIA OF RUPTURE OUT PRINCIPAL AXES}

Criteria given by the equations (2), (5), above, relate to constraints expressed compared to axes of symmetry of the laminate.

When the reference axes are unspecified (fig.1), we must determine the equations of transformation of the constraints. By introducing the assumption of the plane constraints, used within the framework of the theory of the plates aminated, we obtains :

$$
\sigma_{L L}=c^{2} \bullet \sigma_{1}+s^{2} \bullet \sigma_{2}+2 s c \sigma_{6}
$$

$$
\sigma_{T T}=c^{2} \bullet \sigma_{1}+s^{2} \bullet \sigma_{2}-2 s . c . \sigma_{6}
$$

(6.b)

$\sigma_{L T}=\operatorname{s.c}\left(\sigma_{2}-\sigma_{1}\right)+\left(c^{2}-s^{2}\right) \sigma_{6}$

Where

$$
\sigma_{1}=\sigma_{11} ; \quad \sigma_{2}=\sigma_{22} ; \quad \sigma_{6}=\sigma_{12} ;
$$

$c=\cos \theta ; s=\sin \theta$

Thus, it is enough to replace in the equations (5) axial stresses by the equations of transformation of constraints to lead to a general form of the criterion of rupture apart from the principal axes:

$$
\begin{aligned}
& \sigma_{1}^{2} F_{11}+\sigma_{2}^{2} F_{22}+\sigma_{5}^{2} F_{66}+2 \sigma_{1} \sigma_{2} F_{12} \\
& +2 \sigma_{1} \sigma_{6} F_{16} \\
& +2 \sigma_{2} \sigma_{6} F_{26}+\sigma_{1} F_{1}+\sigma_{2} F_{2}+\sigma_{6} F_{6}=1
\end{aligned}
$$

Where the coefficients $F_{i j}$ and $F_{k}$ are a function known and are written in the form:

$$
F_{11}=c^{4} \cdot F_{L L}+s^{4} \cdot F_{T T}+3 s^{2} \cdot c^{2} \cdot F_{L T}
$$

$$
\begin{aligned}
& F_{22}=s^{4} . . F_{L L}+c^{4} F_{T T}+3 s^{2} \cdot c^{2} \cdot F_{L T} \\
& F_{12}=c^{2} \cdot s^{2} \cdot F_{L L}+c^{2} \cdot s^{2} \cdot F_{T T} \\
& +\left(c^{4}+s^{4}-c^{2} \cdot s^{2}\right) F_{L T}
\end{aligned}
$$

$$
\begin{aligned}
& F_{66}=4 c^{2} \cdot s^{2} \cdot F_{L L}+4 c^{2} \cdot s^{2} \cdot F_{T T} \\
& +\left(c^{4}+s^{4}-10 c^{2} \cdot s^{2}\right) F_{L T} \\
& F_{16}=2 c^{3} \cdot s \cdot F_{L L}-24 c \cdot s^{3} \cdot F_{T T} \\
& +3\left(c s^{3}-s c^{3}\right) F_{L T}
\end{aligned}
$$

$$
\begin{aligned}
& F_{26}=2 c \cdot s^{3} \cdot F_{L L}-2 c^{3} \cdot s \cdot F_{T T} \\
& +3\left(c^{3} \cdot s-s^{3} \cdot c\right) \cdot F_{L T}
\end{aligned}
$$


$F_{1}=c^{2} . F_{L L}+. s^{3} \cdot F_{T T}$

(7.8)

$F_{2}=s^{2} \cdot F_{L L}+c^{3} \cdot F_{T T}$

(7.9)

$F_{6}=2 c . s .\left(F_{L L}-F_{T T}\right)$

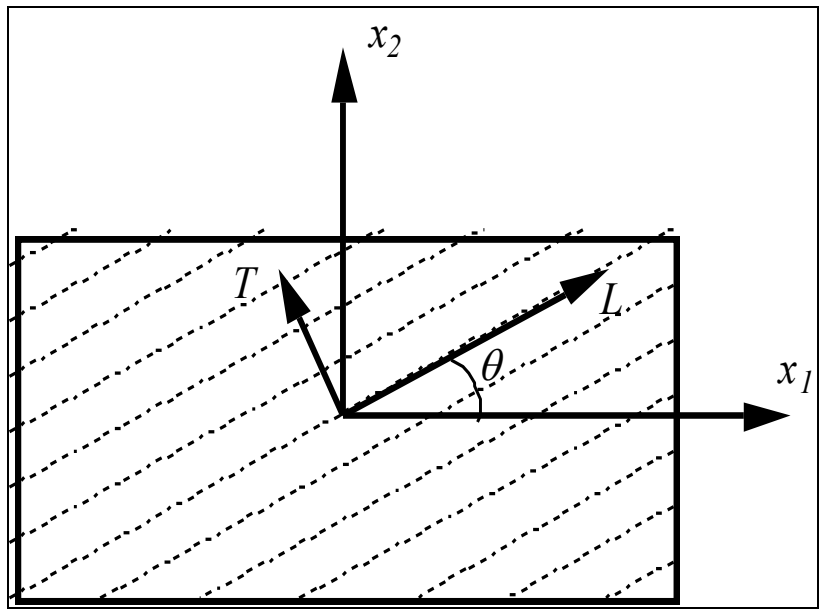

Fig 1: Reference apart from the principal axes.

\section{VALIDATION}

In order to ensure itself of the validity of our results on the level of the criteria of rupture, we studied the case of a laminated plate subjected to a load of traction.

The laminated plate consists of six layers, symmetrical same thicknesses, but different orientations [6,] [9, 11,13, 14, 21].

The geometry of the plate is defined by :II

The Ithicknesses of the elementary layers : $h_{i}=(1 \mathrm{~mm})$

The Ifforientation of fibres in the layers : $\left[0^{0} / 30^{\circ} / 90^{\circ} / 120^{\circ} / 150^{\circ}\right]_{s}$

The mechanical characteristics of the layers are:I $E_{L}=45 G P a . \quad E_{T}=10 G P a, G_{L T}=4.5 G P a$. $v_{L T}=0.31$

The models of rupture are:II

- Test of rupture in traction according to the direction L:II $\sigma_{L L} \leq X=1400 M P a$

- Test of rupture in traction according to the direction $\mathrm{T}: \mathbb{I}$ $\sigma_{T T} \leq Y=40 M P a$

- Test of rupture in shearing: $\sigma_{L T} \leq S=70 \mathrm{MPa}$

To implement our calculations, we introduced into our softwar, a module of calculation which makes it possible to fix an initial load and to check the criterion of rupture adopted for the evaluation of the ultimate stresses in each layer taken individually.

If the limit is reached, we cancel the elastic modules of the layer concerned and we takes again calculation for the remaining layers. That to mean that the eliminated layer does not take part any more in rigidity of the laminate and that with each rupture of layer the behavior total of the laminate is modified and that its rigidity decreases.
If the criterion of rupture is not checked (the ultimate stress is not reached) we increases the load of a step which one fixes initially and we takes again calculations.

The comparison of the results resulting from our calculations to the various criteria of rupture which we chose to apply is presented in table 1.

we notes a very good agreement of the results obtained by our calculations, as well on the level of the values of the ultimate stresses as on the level of the order of the successions of the ruptures.

Indeed, when the load of traction increases, we observes successive ruptures of layers, and this of the first rupture of layers until the rupture of the last layer which coincides with the final rupture of the plate.

Thus, as we presented in the table, the first rupture is carried out transversely in the layers with $90^{\circ}$, the second transverse rupture is localized in the layers with $60^{\circ}$ and $120^{\circ}$, the third rupture by shearing of the layers with $30^{\circ}$ and $150^{\circ}$. The final rupture occurs in the longitudinal direction of the layers with $0^{0}$.

It is noted that the constraints are calculated by the analytical model of the plates and the finite element method. The results which we obtained by the two methods do not show significant differences.

For this reason we limit in table 1 to the presentation of the constraints obtained by the model of the plates (Sinus), which has the advantage of the facility of handling compared to the numerical method of the finite elements.

Table 1: Values of the successive breaking loads

\begin{tabular}{|c|c|c|c|c|}
\hline $\begin{array}{c}\text { Successive } \\
\text { ruptures }\end{array}$ & Referee & TsaiWu & Hill & Hoffmn \\
\hline $\begin{array}{c}1 \text { st rupture with } \\
90^{\circ}\end{array}$ & $95 \mathrm{MPa}$ & $94 \mathrm{MPa}$ & $93 \mathrm{MPa}$ & $90 \mathrm{MPa}$ \\
\hline $\begin{array}{c}2 \mathrm{~d} \text { rupture to } 60^{\circ} \\
\text { and } 120^{\circ}\end{array}$ & $122 \mathrm{MPa}$ & $119 \mathrm{MPa}$ & $117 \mathrm{MPa}$ & $116 \mathrm{MPa}$ \\
\hline $\begin{array}{c}3 \text { rd rupture with } \\
30^{\circ} \text { and } 150^{\circ}\end{array}$ & $238 \mathrm{MPa}$ & $237 \mathrm{MPa}$ & $236 \mathrm{MPa}$ & $233 \mathrm{MPa}$ \\
\hline $\begin{array}{c}\text { final rupture with } \\
0^{\circ}\end{array}$ & $476 \mathrm{MPa}$ & $467 \mathrm{MPa}$ & $473 \mathrm{MPa}$ & $470 \mathrm{MPa}$ \\
\hline
\end{tabular}

\section{CONCLUSION}

$\mathrm{n}$ this study we apply the criteria of rupture to the case of the multi-layer laminates by taking account of the thickness of each layer as well as the orientation of fibers.

And we presented example of application, which it possible to validate our calculations, at the level of the criteria of rupture and the progressive nature of the degradation of a laminated plate highlighted.

The use of these criteria within the framework of a dimensioning of a laminate, with respect to a limiting load can constitute a good approach to optimize the laminated structures. These what us is summoned spirit studied within our laboratory.

Lastly, we showed starting from the examples at least simple, that the coupling of the techniques of experiments and the approach of optimization can lead to the realization of the composite structures very powerful and to lower cost. 


\section{REFERENCES}

[1] J Lemaître, J L Chaboche, Mechanics of solid materials, Dunod, Paris, 1985

[2] R. Hill, The mathematical theory of plasticity, Oxford University Press, London, 1950

[3] O. Hoffman, The brittle strength pf orthotropic material, J. Of, Comp. Materials, V. 1, pp. 200-206, 1967.

[4] V. D. Azzi, S. W. Tsai, Anisotropic strength of component, Experimental Mechanics, V. 5, pp. 286288,1965

[5] S. W. Tsai, E. M. Wu, A general of theory of strength of anisotropic materials, J. Of, Comp. Materials, V. 5, pp. $58-80,1971$

[6] J. M. Berthelot, Matériaux composites : Comportment mécanique et analyse des structures, Masson, Paris, 1992.

[7] N. S. Khot, V. B. Venkayya, C. D. Johnson, V. A. Tischler, Optimisation of fiber reinforced composite structures, Int.J. Solids Structures, V. 9, pp. 12251236,1973 .

[8] T. W. Chou, T. Ishikawa. Analysis and modelling of two dimensional fabric composites. Elsevier, 1989, pp. 209264.

[9] Harald Berger, Sreedhar , Kari, Ulrich Gabbert. An analytical and numerical approach for calculating effective material coefficients of piezoelectric fiber composites. International Journal Of Solids And Structure 42 (2005),pp. 5692-5714

[10] Jorg. Hohe, A direct homogenization approach for determination of the stiffness matrix for microheterogeneous plats with application to sandwich panels . Elsevier composites: part B 34(2003) pp 615-626

[11] S. Alsubari, H. Chaffoui Comparison of the elastic coefficients and Calculation Models of the Mechanical Behavior one- Dimensional Composites IJCSI International Journal of Computer Science Issues, Vol. 8, Issue 5, September 2011,pp 63-67. Online www.IJCSI.org

[12] He Ling-Hui, A linear theory of laminated shells accounting for continuity of displacements and transverse Shear Stress at layer Interfaces, Int. J. Solids Structures, V. 31, N 5, 1994, pp. 613-627.

[13] Z. Hashin, On elastic behaviour of fibre reinforced material of arbitrary transverse plane geometry, J. of the Mech. and Phy. of Solids, V. 13, 1965, pp. 119-126.

[14] X.L. Fanl, Q. Sun, M. Kikuchi Review of Damage Tolerant Analysis of Laminated Composites. Journal of Solid Mechanics Vol. 2, No. 3 (2010) pp. 275-289

[15] P.M. Mohite, C.S. Upadhyay, Region-by-region modeling of laminated composite plates. Elsevier Computers and Structures 85 (2007) 1808-1827

[16] Andrzej Katunin , Characterization of damage evolution during fatigue of composite structures accompanied with self-heating effect by means of acoustic emission, https://www.researchgate.net/publication/324134115 All content following this page was uploaded by Andrzej Katunin on 19 July 2018.

[17] Mehdi Yasaee, Lawrence Bigg, Galal Mohamed, Stephen R. Hallett , Influence of Z-pin embedded length on the interlaminar traction response of multi-directional composite laminates, Materials \& Design, Volume 115, 5 February 2017, pp. 26-36

[18] B. Sabuncuoglu, S.A. Tabatabaei and S.V. Lomov, Analysis Of Fiber/Matrix Interface Debonding In Steel Fiber Composites Under Transverse Loading, ECCM18 18th European Conference on Composite Materials Athens, Greece, 24-28th June 2018

[19] Lp khoroshun , En shikula , micromechanics of long term damage of particulate composites with unlimited miicrodurability , international Applied Mechanics 44(11), 1204- 1212,2008

[20] A. Ríos and A. Martín-Meizoso , Micromechanical Model of Interface Between Fibre and Matrix of Metal Matrix Composite Reinforced with Continuous Fibre , Advanced materials research Vol. 59 (2009)pp 158-163

[21] S. Alsubari, H. Chaffoui, Modeling and Analysis of Laminated Composite Structures, International Journal of Computer Applications (0975 - 8887) Volume 50 No.13, July 2012

[22] Roman Vodikaa,, Jozef, Kšinan, Interfacial Debonds in Unidirectional Fibre-Reinforced Composites Exposed to Biaxial Loads, Procedia Engineering 190 ( 2017 ) 433 440

[23] Cyril Bordreuil , christian Hochard , Finite element computation of woven ply laminated composite structures up to rupture , Applied composite Materials $11(3), 127-143,2004$ 